\section{Clinical, diagnostic and immunological characteristics of patients with possible neuroborreliosis without intrathecal Ig-synthesis against Borrelia antigen in the cerebrospinal fluid}

\author{
Magnus Vrethem,1 Mona Widhe,2,3,4 \\ Jan Ernerudh,2,4 Ulf Garpmo, 5 \\ Pia Forsberg 3 \\ 1Division of Neurology and \\ Neurophysiology, Department of Clinical \\ and Experimental Medicine, University \\ Hospital, Linköping; 'Division of Clinical \\ Immunology, Department of Clinical and \\ Experimental Medicine, University \\ Hospital, Linköping; 3Division of \\ Infectious Diseases, Department of \\ Clinical and Experimental Medicine, \\ University Hospital, Linköping; \\ 4Unit for Autoimmunity and Immune \\ regulation, Faculty of Health Sciences, \\ University of Linköping; ${ }^{5}$ Department \\ of Microbiology, Kalmar Hospital, \\ Sweden
}

\section{Abstract}

The diagnosis of neuroborreliosis is not always straightforward. Intrathecal immunoglobulin (Ig) synthesis against Borrelia antigen may not be detected, at least early in the disease course. Also other neurological and infectious diagnoses have to be considered. We have studied patients with clinical possible neuroborreliosis without intrathecal Ig synthesis against Borrelia antigen in the cerebrospinal fluid (CSF) $(n=17)$. Diagnosis was based on typical clinical history and at least one of the following findings; mononuclear leucocytosis in the CSF $(n=4)$; typical erythema migrans $>5 \mathrm{~cm}$ in diameter in relation to debut of symptoms $(n=8)$; prompt clinical response to antibiotic teratment $(n=14)$. Also other possible diagnoses had to be excluded. Seventeen patients first investigated because of suspected neuroborreliosis but later confirmed with other diagnoses were used as controls. All patients had a lumbar puncture. Borrelia specific IFN- $\gamma$ and IL-4 secretion was investigated in peripheral blood (PBL) and CSF with an ELISPOT assay. Polymerase chain reaction (PCR) was used to reveal any Borrelia antigen in the CSF. Six of 17 patients with possible neuroborreliosis showed high IFN- $\gamma$ secretion in peripheral blood, otherwise we found no statistically sig- nificant differences between the groups. PCR did not reveal any Borrelia antigen in CSF. The diagnosis and treatment of possible but not confirmed neuroborreliosis is a clinical challenge. The clinical response to treatment may be the best option in these cases.

\section{Introduction}

Borrelia can affect many organ systems, i.e. skin, heart, joints and the nervous system. ${ }^{1}$ Clinical symptoms are variable and depend on the subtype of Borrelia. Borrelia burgdorferi sensu latu typically cause arthritis. Borrelia garinii mainly causes neurological symptoms ${ }^{2}$ whereas Borrelia afzelii give rise to the erythema chronicum migrans, common for all three subtypes.

The neurological symptoms are not specific for neuroborreliosis but may occur in many other neurological diseases. ${ }^{3}$ This makes the clinical diagnosis difficult unless there is a preceding erythema migrans. Also laboratory parameters to diagnose neuroborrelia are uncertain because of a late and inconsistent occurrence of antibodies against Borrelia. Intrathecal synthesis of Borrelia antibodies is of diagnostic importance 4 and so is mononuclear leucocytosis in the cerebrospinal fluid (CSF) and signs of blood-brain barrier damage. 5 Polymerase chain reaction (PCR) has been utilised in several studies but is not routinely recommended due to the limited clinical sensitivity of this method.6,7 When the diagnosis of neuroborreliosis is confirmed or highly likely treatment with antibiotics is initiated. Usually neurological symptoms are reduced and eventually disappear. Some patients, however, experience reappearing or persistent neurological symptoms some time after the treatment. ${ }^{1}$ It is not clear if these late-symptoms are related to persistent symptoms of an Borrelia infection or if they are caused by other neuroimmunological reactions or both. Persistent symptoms may also be due to irreversible damage of the nervous system. The purpose of our study was to investigate patients with clinical possible neuroborreliosis but lacking intrathecal synthesis of antibodies against Borrelia antigen. In clinical practice there is a need for other methods to discriminate true neuroborreliosis patients from other with mimicking symptoms and laboratory parameters. We aimed at looking for levels of the type 1 cytokine IFN- $\gamma$ and the type 2 cytokine IL4 in CSF and blood.

\section{Materials and Methods}

\section{Patients and controls}

Clinical and laboratory supported definite
Correspondence: Magnus Vrethem, Division of Neurology and Neurophysiology, University Hospital, S-581 85 Linköping, Sweden.

Tel. +46.101032048 - Fax. +46.101034541.

E-mail: magnus.vrethem@lio.se

Key words: Borrelia; neuroborreliosis; neurological symptoms; cerebrospinal fluid; cytokines; chronic

Acknowledgements: we would like to thank Christina Ekerfelt for practical help throughout the study. Financial support from The Association of Neurologically Disabled and Östergötlands Läns Landsting was given.

Received for publication: 12 February 2011.

Revision received: 24 March 2011.

Accepted for publication: 28 March 2011.

This work is licensed under a Creative Commons Attribution 3.0 License (by-nc 3.0).

(C) Copyright M. Vrethem et al., 2011 Licensee PAGEPress, Italy

Neurology International 2011; 3:e2 doi:10.4081/ni.2011.e2

neuroborreliosis ${ }^{8}$ is characterized as; typical clinical neurological symptoms (meningitis, cranial neuritis, or radiculitis), CSF mononuclear leucocytosis and intrathecal synthesis of Borrelia specific antibodies (Daco kit for detection of anti-flagellin antibodies in serum and CSF was used). ${ }^{9}$ We identified a subgroup of patients with typical neurological symptoms without intrathecal synthesis of Borrelia specific antibodies ( $n=17,9$ women and 8 men, mean age 50.5 years) but with intrathecal leucocytosis $(n=4)$, erythema migrans in relation to symptoms $(n=8)$ and/or response to antibiotic treatment $(n=14)$, referred to as possible neuroborreliosis. Eleven of the 17 patients with possible neuroborreliosis, however, showed positive serology for IgG and/or IgM against Borrelia in serum. Seventeen patients first investigated because of suspected neuroborreliosis but later confirmed with other diagnoses were used as controls (8 women and 9 men, mean age 44.5 years). Characteristics of the patients are shown in Table 1.

\section{Preparation of mononuclear cells \\ from blood and cerebrospinal fluid}

Blood (heparinized) and CSF samples were obtained from patients with suspected NB. Peripheral blood mononuclear cells (PBMC) were separated by gradient centrifugation on Lymphoprep ${ }^{\circledR} \quad$ (Medinor AB, Stockholm, Sweden) at $400 \mathrm{~g}$ for 30 minutes in room temperature, according to Bøyum. ${ }^{10}$ Cells were counted under a phase-contrast microscopy using a Bürker chamber, and the lymphocyte concentration was adjusted to $1 \times 106 / \mathrm{mL}$. CSFcells were counted using phase-contrast 
Table 1. Characterization of patients with possible neuroborreliosis without intrathecal Ig-synthesis against Borrelia antigen in the cerebrospinal fluid and controls with other diagnosesPat.

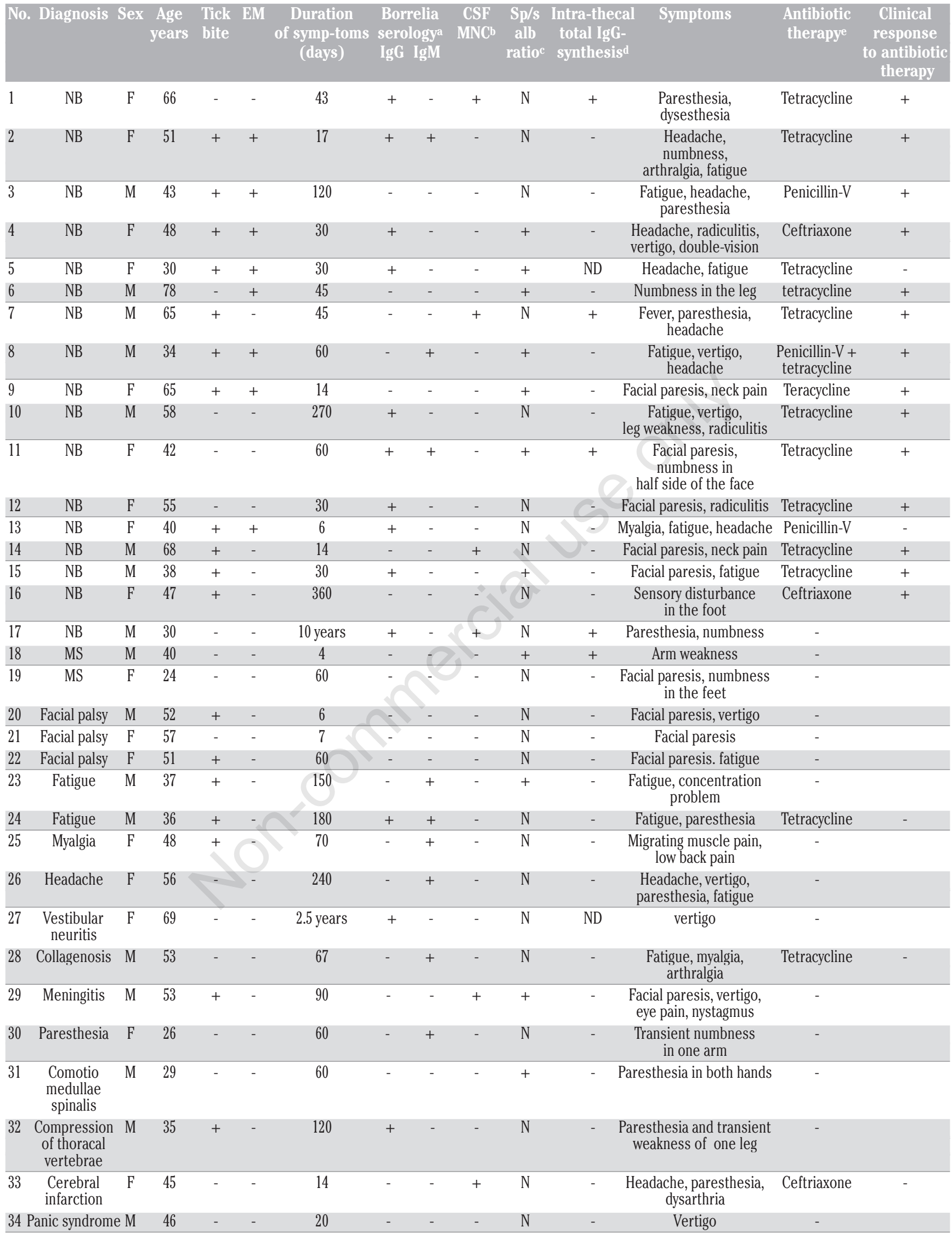

MS, multiple sclerosis; EM, erythema migrans in relation to symptoms; N, normal; ND, not done; F, female, M, male. Duration of symptoms in days if not stated otherwise. a+= patients positive in the Borrelia ELISA for IgG or IgM in blood. bMononuclear leucocytosis in the cerebrospinal fluid $\geq 5 \times 106 / \mathrm{L}$. ${ }^{\mathrm{S} S p i n a l} / \mathrm{serum}$ albumin ratio (Sp/s alb ratio) (+=elevated). ${ }^{\mathrm{d}}+=\mathrm{IgG}$ index $\geq 0.7$. ${ }^{\mathrm{e} T e t r a c y c l i n e ~ w a s ~ g i v e n ~ a s ~ a n ~ o r a l ~ d o s e ~ o f ~} 200$ mg daily for 14 days; penicillin- $2 \mathrm{gr}$ orally daily for 10 days; ceftriaxone $2 \mathrm{gr}$ intravenously daily for 14 days. 
microscopy, by use of a Jessen chamber, before undergoing 10 minutes of centrifugation at $200 \mathrm{~g}$ at $4^{\circ} \mathrm{C}$, followed by gentle resuspension in cell culture medium (TCM).

\section{Preparation of outer surface pro- tein fraction Borrelia antigen}

An outer surface protein (osp) enriched fraction (OF), mainly containing OspA and $0 \mathrm{spB}$, was prepared from of Borrelia garinii strain Ip90, as previously described.11-13 OF stimulation of PBMC and CSF-MNC was shown in adults to discriminate between patients with neuroborreliosis and patients with other neurological diseases as well as healthy controls in the IFN $\gamma$ and IL 4-ELISPOT-assay.14,15

\section{ELISPOT for analysis of IL-4 and IFN- $\gamma$ producing cells}

The ELISPOT assay, which was originally described by Czerkinsky et al., ${ }^{16}$ was used for analysis of Borrelia (OF)-stimulated and unstimulated cytokine secreting cells. The method was slightly modified and optimized for IL- 4 and IFN- $\gamma$ spots as described previously. ${ }^{11-13}$ The spots were counted under a dissection microscope. One spot resembles one cytokine secreting cell.

\section{DNA extraction and polymerase chain reaction amplification}

A QIAamp tissue kit (Qiagen) was used for DNA extraction according to the protocol of the manufacturer. Samples were eluted with $50 \mu \mathrm{L}$ of $\mathrm{AE}$ buffer and stored in $-20^{\circ} \mathrm{C}$. For detection of Borrelia antigen the 16S rRNA sequence was amplified by a nested PCR. Primers16S-F and $16 \mathrm{~S}-\mathrm{R}$ and LD1 and LD2 were used in the amplification. The products were visualized by electrophoresis in a $1.5 \%$ agarose gel stained with ethidium bromide. A negative control and a positive control were included in all PCR runs. The method is previously described by us. 17

\section{Data handling and statistics}

The mean of triplicates or duplicates was used in the analysis of cytokine results. To determine Borrelia specific secretion, the number of spots in the unstimulated wells was subtracted from the number in the OF-stimulated wells. For CSF-cells, data were recalculated to the number of spots/100 000 lymphocytes. For comparison of cytokine secreting cell counts between multiple groups or intervals, KruskalWallis was used as a pre-test, and MannWhitney $\mathrm{U}$ test as post-hoc. $\mathrm{P}<0.05$ were considered significant.

The present study was approved by the ethics committee of the Faculty of Health Sciences at Linköping University (Linköping, Sweden). Informed consent was obtained from each of the patients included in the study.

\section{Results}

Six of 17 patients with possible neuroborreliosis had the highest IFN- $\gamma$ secretion in peripheral blood upon Borrelia antigen stimulation. The median values between the groups were not statistically significant, however. We found no differences in IL- 4 or IFN- $\gamma$ secretion in the CSF (Table 2 and Figure 1). PCR did not reveal any Borrelia antigen in CSF.

Borrelia serology in blood seems unspecific and could mirror an earlier infection or in case of IgM antibodies be an unspecific reaction (10 had IgG and $3 \operatorname{IgM}$ of the neuroborreliosis patients and 3 had IgG and 6 IgM of the controls). Also mononuclear leucocytosis in the CSF, blood-brain barrier damage, and intrathecal immunoglobulin synthesis were seen in both possible neuroborreliosis patients and controls with other diagnoses. In the control group mononuclear leucocytosis in the CSF was seen in one patient with viral meningitis and one with cerebral infarction; blood-brain barrier damage in one patient with multiple sclerosis (MS), fatigue, viral meningitis and comotio medullae spinalis, respectively; intrathecal immunoglobulin synthesis in one MS patient.

Symptoms are often non-specific such myalgia, paresthesia, fatigue and headache and were reported equally in the possible neuroborreliosis patients and controls with other diagnoses. In the control group 3 patients with facial palsy (Bells palsy) and 3 with unspecific fatigue or myalgia and 1 with transient paresthesia and 1 with tension headache without preceding erythema migrans or mononuclear leucocytosis in CSF were included. Otherwise the diagnoses in the control group were diverse but specific (Table 1).

The most reliable clinical feature of a possible neuroborreliosis was a preceding erythema migrans in 8 out of 17 patients in contrast to none of the 17 controls. Tick bite did not discriminate between the groups.

Clinical response to antibiotic treatment was seen in 14 out of 16 treated patients with possible neuroborreliosis (12 were treated with tetracycline $200 \mathrm{mg}$ orally for 14 days; 2 with ceftriaxone $2 \mathrm{gr}$ iv for 14 days; 3 with penicillin-V 2 gr orally for 10 days, one of which also treated with tetracycline) and could be taken as an indication of at least an infectious etiology. Patient no 5 and 13 in table 1 did not respond to treatment. The first patient had persistent headache and fatigue after tetracycline treatment and the second patient was treated early after debut of symptoms (6 days) and received only penicillin-V orally. In the control group 3 patients were treated on the early suspicion of neuroborreliosis (2 with tetracycline and one with ceftriaxone) but later confirmed with other diagnoses.

Patient no 10, 12, 15, and 16 were judged as possible neuroborreliosis (Table 1) because of typical neurological symptoms (2 radiculitis, 1 facial palsy and one with a radicular distribution of sensory disturbance in the foot) and prompt response to antibiotic treatment. These 4 patients, however, did not report any erythema migrans or had any CSF findings (except one with a non-specific elevated spinal/serum albumin-ratio).

One patient (no 17) judged as possible neuroborreliosis was not treated because of longlasting symptoms (10 years). These patients are referred to as post-Lyme disease syndrome (PLDS) and therapy has no clear impact of the symptoms. Three of our patients with possible neuroborreliosis had their symptoms for more than 6 months, often classified as late neuroborreliosis, ${ }^{18}$ but the majority of our patients had early possible neuroborreliosis.

\section{Discussion}

The most common diagnostic tool to the diagnosis of neuroborreliosis is ELISA analysis for antibodies against the Borrelia antigen. We

Table 2. Net secretion of IFN- $\gamma$ and IL- 4 spots in patients with possible neuroborreliosis and patients with other diagnoses.

\begin{tabular}{lcccc} 
& Diagnosis & N & Mean rank & Pa \\
Net IFN- $\gamma$ in PBL & Neuroborreliosis & 15 & 16.7 & 0.12 \\
Spots/100 000 cells & Other diagnosis & 13 & 11.9 & \\
& Total & 28 & & \\
Net IL-4 in PBL & Neuroborreliosis & 15 & 15.9 & 0.33 \\
Spots/100 000 cells & Other diagnosis & 13 & 12.9 & \\
& Total & 28 & & \\
\hline Net IFN- $\gamma$ in CSF & Neuroborreliosis & 12 & 12.10 & 0.72 \\
Spots/100 000 cells & Other diagnosis & 10 & 11.0 & \\
& Total & 22 & & \\
Net IL-4 in CSF & Neuroborreliosis & 8 & 6.4 & 0.54 \\
Spots/100 000 cells & Other diagnosis & 3 & 5.0 & \\
& Total & 11 & &
\end{tabular}

aMann-Whitney 
used the Daco kit for detection of anti-flagellin antibodies in serum and CSF, previously shown to be a sensitive and specific test. ${ }^{19}$ None of the 17 patients with possible neuroborreliosis had antibody titers against the flagellin antigen in CSF, but 11 had elevated IgG or IgM titers in serum. PCR did not reveal any Borrelia antigen in CSF and the method is often regarded as of low sensitivity, especially in blood and in late neuroborreliosis cases. ${ }^{18}$

The earliest indication of Borrelia infection is usually erythema migrans. ${ }^{20}$ Eight patients (47\%) with possible neuroborreliosis had noticed an erythema migrans in relation to symptoms in the present study. Only 15 (14\%) of 106 patients with definite neuroborreliosis had noted an erythema migrans in a previous study. ${ }^{21}$ A typical erythema migrans with a diameter of $>5 \mathrm{~cm}$ in conjunction with symptoms is usually regarded as more or less proof of a Borrelia infection.22

Leucocytosis in the CSF is a common finding in neuroborreliosis but may be a finding in many other infectious or inflammatory diseases. All patients were lumbar punctured and 4 of 17 showed leucocytosis in the present study. Also one patient with cerebral infarction and one with viral meningitis showed leucocytosis. We have previously shown that 85 of 106 patients with ELISA positive neuroborreliosis had leucocytosis $(5-2330 \times 106 / \mathrm{L})$ in the CSF. It is possible that the lower number of patients with leucocytosis in the CSF in the present study reflects a weak immune response against the Borrelia antigen. Another possibility is that the lumbar puncture was taken too early in the disease process. Only 6 were taken one month or earlier after the debut of symptoms, however. A diagnosis and treatment of neuroborreliosis is not always straightforward and proposed guidelines for the management of this has recently been published. 18,23

We previously showed a compartmentalized interferon-gamma (IFN- $\gamma$ ) response in the central nervous system (CNS) in patients with neuroborreliosis ${ }^{15}$ and that an IFN- $\gamma$ predominated response was associated with a good prognosis in asymptomatic sero-positive individuals. ${ }^{24}$ In the present study we did not find any significant difference in IFN- $\gamma$ or IL-4 production in the CSF, however. Recently elevated levels of the B-lymphocyte attracting chemokine (CXCL13) in the CSF in early neuroborreliosis patients have been found. 25 It has also been seen in the CSF of multiple sclerosis patients ${ }^{26}$ making the specificity of the test uncertain. Most of our patients with possible neuroborreliosis were treatment with doxycycline reported to have a downregulatory effect on proinflammatory cytokines. ${ }^{27}$ If this has any significant relevance is uncertain and furthermore little is known about the effects on the IFN- $\gamma$ or IL-4 responses.

The diagnosis and treatment of possible but not confirmed neuroborreliosis is a clinical challenge. Even though we found the highest IFN- $\gamma$ levels in 6 out of 17 patients with possible neuroborreliosis in blood, the test is unspecific and may be elevated also in other inflammatory conditions. Further improvements in diagnostic tests are warranted to establish early diagnosis and to avoid late complications. The clinical picture may raise the suspicion of neuroborreliosis, which can be further strengthened by a preceding erythema migrans and/or elevated mononuclear cell count in the cerebrospinal fluid. A pragmatic approach has been put forward in patients with

\section{PBL OF specific IFN-gamma}
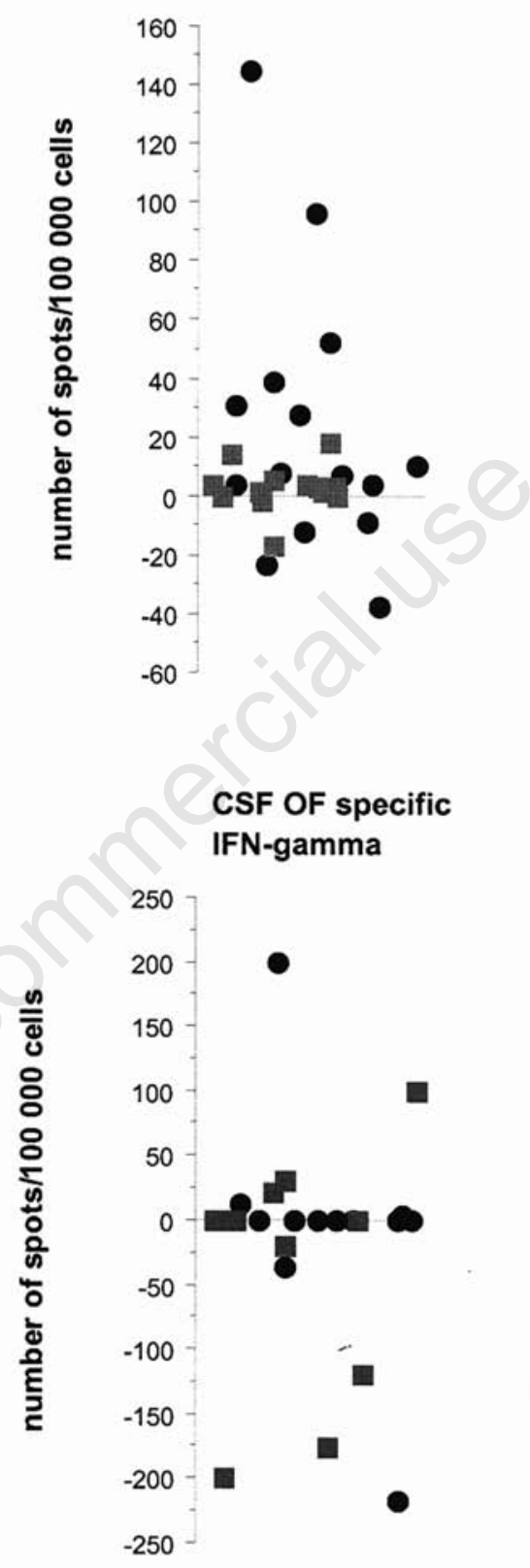

Possible neuroborreliosis
PBL OF specific IL-4

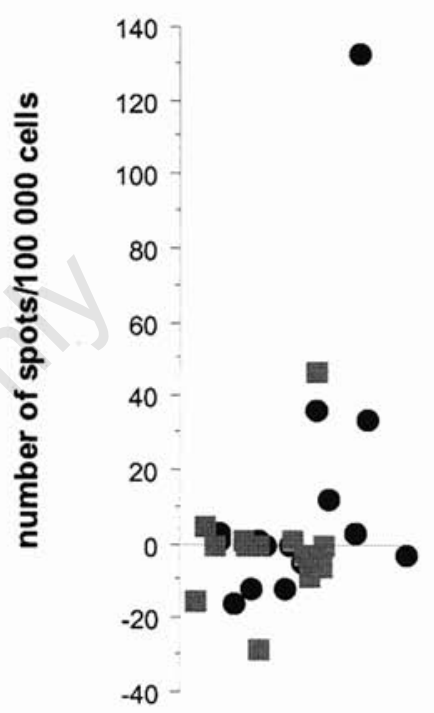

CSF OF specific IL-4

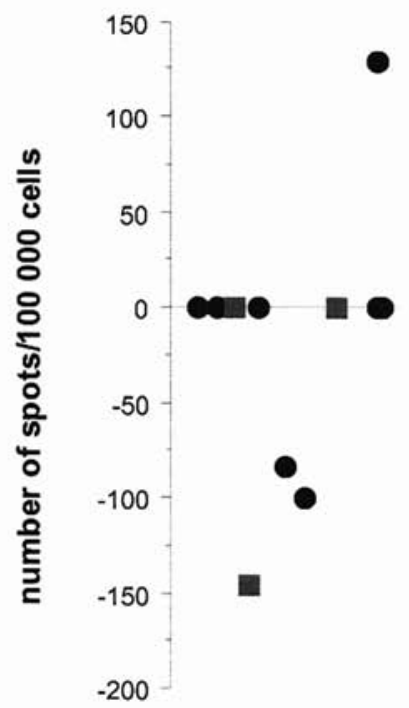

Figure 1. Number of Borrelia specific IFN- $\gamma$ and IL-4 secreting cells per 100.000 lymphocytes in cerebrospinal fluid and peripheral blood in patients with probable neuroborreliosis and patients with other diagnoses as measured by ELISPOT. The Borrelia specific secretion was obtained by subtracting the number of spots in unstimulated wells from the number in Borrelia OF-stimulated wells. 
possible neuroborreliosis, namely a favorable response to antibiotic treatment. ${ }^{28}$ Our study also indicates that the clinical response to treatment may be the best option in patients with possible neuroborreliosis.

\section{References}

1. Treib J, Frenandez A, Haass A, et al. Clinical and serologic follow-up in patients with neuroborreliosis. Neurology 1998;51:1489-91.

2. Haas A. Lyme neuroborreliosis. Current Opinion in Neurology 1998;11:253-8.

3. Stiernstedt G, Gustavsson R, Karlsson M, et al. Clinical manifestations and diagnosis of neuroborreliosis. Ann NY Acad Sci 1988;539:46-55.

4. Hansen K, Lebech AM. Lyme borreliosis: a new sensitive diagnostic assay for intrathecal synthesis of Borrelia burgdorferi-specific immunoglobulin G, A and M. Ann Neurol 1991;30:197-205.

5. Kaiser R. Neuroborreliosis. J Neurol 1998;245:247-55.

6. Keller TJ, Halperin JJ, Whitman M. PCR detection of Borrelia burgdorferi DNA in cerebrospinal fluid of Lyme neuroborreliosis patients. Neurology 1992;42:32.

7. Tumani H, Nölker G, Reiber H. Relevance of cerebrospinal fluid variables for early diagnosis of neuroborreliosis. Neurology 1995;45:1663-70.

8. Halperin J, Logigian E, Finkel M, Pearl R. Practice parameters for the diagnosis of patients with nervous system Lyme borreliosis (Lyme disease). Neurology 1996; 46:619-27.

9. Skogman BH, Croner S, Nordwall M, et al. Lyme neuroborreliosis in children: a prospective study of clinical features, prognosis, and outcome. Pediatr Infect Dis J 2008;27:1089-94.

10. Bøyum, A. Isolation of mononuclear cells and granulocytes from human blood. Isolation of monuclear cells by one cen- trifugation, and of granulocytes by combining centrifugation and sedimentation at 1 g. Scand J Clin Lab Invest Suppl 1968;97:77-89.

11. Magnarelli LA, Anderson JF, Barbour AG. Enzyme-linked immunosorbent assays for Lyme disease: reactivity of subunits of Borrelia burgdorferi. $\mathrm{J}$ Infect Dis 1989;159:43-9.

12. Bergström S, Sjöstedt A, Dotevall L, et al. Diagnosis of Lyme borreliosis by an enzyme immunoassay detecting immunoglobulin $\mathrm{G}$ reactive to purified Borrelia burgdorferi cell components. Eur J Clin Microbiol Infect Dis 1991;10:422-7.

13. Widhe M, Jarefors S, Ekerfelt $\mathrm{C}$, et al. Borrelia specific IFN- $\gamma$ and IL-4 secretion in CSF and blood during the course of Human Lyme Borreliosis: relation to clinical outcome. J Infect Dis 2004;189:188191.

14. Forsberg P, Ernerudh J, Ekerfelt C, et al. The outer surface proteins of Lyme disease borrelia spirochetes stimulate T cells to secrete interferon-gamma (IFNgamma): diagnostic and pathogenic implications. Clin Exp Immunol 1995;101:45360 .

15. Ekerfelt C, Ernerudh J, Bunikis J, et al. Compartmentalization of antigen specific cytokine responses to the central nervous system in CNS borreliosis: secretion of IFN-gamma predominates over IL-4 secretion in response to outer surface proteins of Lyme disease Borrelia spirochetes. J Neuroimmunol 1997;79:155-62.

16. Czerkinsky C, Andersson G, Ekre HP, et al. Reverse ELISPOT assay for clonal analysis of cytokine production. I. Enumeration of gamma-interferon-secreting cells. $\mathrm{J}$ Immunol Methods 1988;110:29-36.

17. Fraenkel CJ, Garpmo U, Berglund J. Determination of novel Borrelia genospecies in Swedish Ixodes ricinus ticks. J Clin Microbiol 2002;40:3308-12.

18. Mygland A, Ljøstad U, Fingerle V, et al; European Federation of Neurological Societies. EFNS guidelines on the diagno- sis and management of European Lyme neuroborreliosis. Eur J Neurol 2010;17:816.

19. Ekerfeldt C, Ernerudh J, Forsberg P, et al. Lyme Borreliosis in Sweden - diagnostic performance of five commercial Borrelia serology kits using sera from well-defined patient groups. APMIS 2004;112:74-8.

20. Halperin JJ. Nervous system lyme disease: diagnosis and treatment. Rev Neurol Dis 2009;6:4-12.

21. Vrethem M, Hellblom L, Widlund M, et al. Chronic symptoms are common in patients with neuroborreliosis - a questionnaire follow-up study. Acta Neurol Scand 2002;106:205-8.

22. Dandache P, Nadelman RB. Erythema migrans. Infect Dis Clin North Am 2008;22:235-60.

23. O'Connell S. Lyme borreliosis: current issues in diagnosis and management. Curr Opin Infect Dis 2010;23:231-5.

24. Ekerfelt C, Forsberg P, Svenvik M, et al. Asymptomatic Borrelia-seropositive individuals displayed the same incidence of Borrelia-specific interferon-gamma (IFN$\gamma$ )-secreting cells in blood as patients with clinical Borrelia infection. Clin Exp Immunol 1999;115:498-502.

25. Ljöstad U, Mygland A. CSF B-lymphocyte chemoattractant (CXCL13) in the early diagnosis of acute Lyme neuroborreliosis. J Neurol 2008;255:732-7.

26. Sellebjerg F, Börnsen L, Khademi M, et al. Increased cerebrospinal fluid concentrations of the chemokine CXCL13 in active MS. Neurology 2009;73:2003-10.

27. Labro MT. Interference of antibacterial agents with phagocyte functions: immunomodulation or "immuno-fairy tales" ? Clin Microbiol Rev 2000;13:615-50.

28. Blanc F, Jaulhac B, Fleury $M$, et al. Relevance of the antibody index to diagnose Lyme neuroborreliosis among seropositive patients. Neurology 2007;69: 953-8. 